\title{
Control of Yaw Disturbance Using Fuzzy Logic Based Yaw Stability Controller
}

\author{
S. Krishna, S. Narayanan, and S. Denis Ashok \\ School of Mechanical and Building Sciences, VIT University, Vellore 632-014, India \\ Correspondence should be addressed to S. Krishna; krishna.s@vit.ac.in
}

Received 31 August 2013; Accepted 2 December 2013; Published 4 February 2014

Academic Editor: Shinsuke Hara

Copyright ( 2014 S. Krishna et al. This is an open access article distributed under the Creative Commons Attribution License, which permits unrestricted use, distribution, and reproduction in any medium, provided the original work is properly cited.

\begin{abstract}
Yaw stability is an important consideration for the vehicle directional stability and handling behavior during emergency maneuvers. In order to maintain the desired path of the vehicle, in presence of disturbances due to cross wind, different road conditions, and tire deflections, a fuzzy logic based yaw stability controller is proposed in this paper. Proposed control system receives yaw rate error, steering angle given by the driver, and side slip angle as inputs, for calculating the additional steering angle as output, for maintaining the yaw stability of the vehicle. As the side slip angle cannot be measured directly in a vehicle, it was estimated using a model based Kalman observer. A two-degrees-of-freedom vehicle model is considered in the present work. The effect of disturbance on yaw rate and yaw rate error of the vehicle is simulated for sinusoidal, step maneuver and compared with the existing fuzzy control system which uses two inputs such as steering angle and yaw rate. The simulation results show better performance of the proposed fuzzy based yaw controller as compared with existing control system. Proposed fuzzy based yaw stability controller can be implemented in steer-by-wire system for an active front steering of a road vehicle.
\end{abstract}

\section{Introduction}

The modern automotive researches are focusing on autonomous and semiautonomous ground vehicle to improve stability and prevent the vehicles from spinning, drifting out, and rolling over. Yaw stability is one of the most significant aspects of the vehicle safety and yaw stability control can avoid dangerous, undesirable behaviors of the vehicles in extreme maneuvers. Active front steering control is one of the most practical approaches for yaw stability control in which a correction of steering angle is added to the driver's steering input, when the desired yaw rate of the vehicle fluctuates due to external disturbances. Steering actuated yaw stability control gives maximum benefit with steer-bywire systems, as mechanical linkages between the steering wheel and road wheels are replaced with electronic actuators, which motivates easier implementation of active front wheel steering control based on feedback.

Many research works are focusing on developing yaw stability control systems with steer-by-wire systems. Ackermann (1994) suggests a steering control system which achieves robust decoupling of the vehicle's for yaw stabilization and
(1997) yaw disturbance can also be minimized [1, 2]. Güenç, discusses the robust yaw stability control based on active front steering for road vehicle and its performance was verified using hardware in loop simulation [3]. Canale et al. suggest an internal model control and sliding mode control approaches for yaw stability control were designed and their performances are verified using simulation studies [4]. Falcone et al. proposed direct yaw moment control method using in wheel motor control for enhancing vehicle stability [5]. Oncu proposed a disturbance observer based yaw stability controller for light commercial vehicle [6]. Nam developed a control system consisting of a steering angle disturbance observer and proportional integral type tracking controller for robust control of yaw stability of an electric vehicle [7]. A proportional integral and derivative (PID) control system is developed for yaw stability control based on road wheel steer angel as control input. Bianchi et al. proposed an adaptive integrated control system using active front steering and rear torque vectoring [8]. Arabi and Behroozi proposed an integrated vehicle dynamics control system based on the combination of active front steering (AFS) and active rear differential (ARD) for yaw rate stability 
of the vehicle [9]. Hakima and Ameli presented an integrated control approach for four-wheel steering of automotive system [10]. These control techniques are complex and the implementation is difficult, as they require coordinated control of vehicle subsystems for performing the desired control function.

Many researchers have applied fuzzy logic approach for developing active front wheel steering based yaw stability control systems. Boada et al. applied fuzzy logic approach for developing a control system to maintain the targeted values of yaw rate and side slip angle for a vehicle [11]. Sharif-Ul Hasan and Anwar designed a yaw stability controller of a vehicle via steer-by-wire system [12]. Ko et al. proposed the fuzzy logic based yaw stability controller based on the input parameters such as yaw rate and steering angle [13]. Goodarzi et al. designed a fuzzy controller based on yaw rate error, side slip angle, and a lateral acceleration of the vehicle for calculating the corrected steering angles of the front inner and outer wheels of the vehicle [14]. Ghosh et al. proposed fuzzy logic based active yaw control algorithm, which receives yaw rate and steering angle as inputs for generating additional steering angle [15]. Geng et al. used an observer based approach for estimating the body slip angle of a vehicle [16].

This paper presents fuzzy based yaw stability control system which requires three input parameters which are yaw rate error, steering angle, and vehicle body side slip angle of the vehicle, for maintaining yaw rate of the vehicle by generating additional steering angle to the front wheels of the vehicle. In distinction to the existing methods, the proposed fuzzy control system uses a Kalman filter based observer for body side slip angle estimation of the vehicle for better maintenance of yaw rate error. Simulation results are presented for analyzing the performance of proposed approach.

\section{Vehicle Dynamics Model}

In the present work, a three-degrees-of-freedom (DOF) vehicle model is considered for analyzing lateral dynamics of the vehicle, which is adapted from [1]. The vehicle dynamics model used in present work is shown in Figure 1. This model describes the lateral dynamics such as yaw rate $(\dot{\psi})$, lateral acceleration $\left(a_{y}\right)$, tire forces on front, and rear wheels.

Lateral acceleration is written as follows:

$$
a_{y}=\ddot{y}+V_{x} \dot{\psi},
$$

$\ddot{y}$ refers to acceleration of the vehicle in $Y$-direction and $V_{x}$ refers the longitudinal velocity of the vehicle.

The equation of lateral translation motion is

$$
m a_{y}=m\left(\ddot{y}+V_{x} \dot{\psi}\right)=F_{y f}-F_{y r} .
$$

The terms $F_{y f}$ and $F_{y r}$ describe the respective tire forces on front and rear wheels in " $Y$ " directions of the vehicle. " $m$ " refers to the mass of vehicle and " $V_{x}$ " refers the longitudinal velocity of the vehicle. Yaw angle of the vehicle, which describes the orientation of the vehicle, is shown in Figure 2.

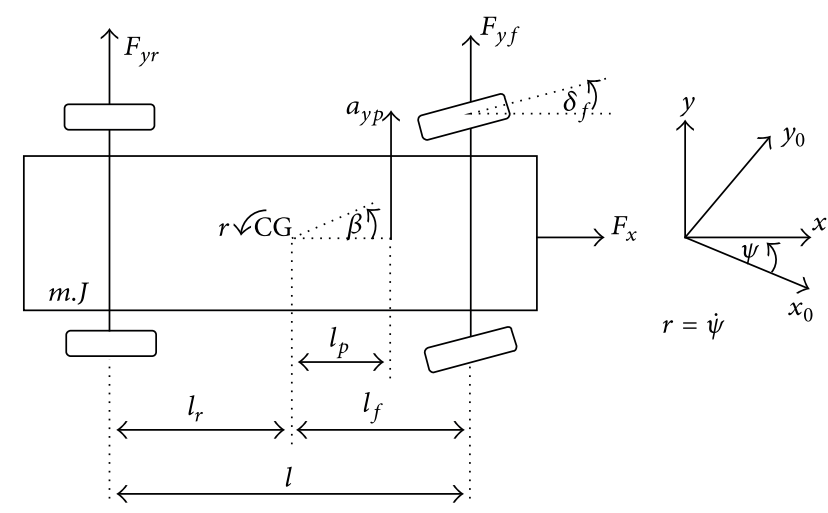

FIGURE 1: 2-DOF vehicle model, [1].

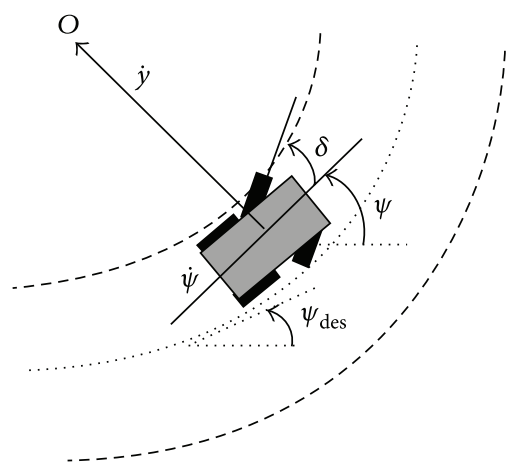

Figure 2: Lateral vehicle dynamics.

The actual yaw moment of the vehicle with external moment $\left(M_{d}\right)$ due to disturbance is given by

$$
I_{z} \ddot{\psi}=l_{f} F_{y f}-l_{r} F_{y r}+M_{d} .
$$

Lateral tire forces of front and rear wheels of the vehicle are as follows:

$$
\begin{gathered}
F_{y f}=2 C_{a f}\left(\delta-\theta_{v f}\right), \\
F_{y r}=2 C_{a r}\left(-\theta_{v r}\right) ;
\end{gathered}
$$

$l_{f}$ and $l_{r}$ refer to the longitudinal distance from CG to front and rear tires, respectively, as shown in Figure 1. Here $C_{a f}$ and $C_{a r}$ represent the cornered force of front and rear wheels, respectively. $\theta_{v f}$ and $\theta_{v r}$ are the velocity angles of the front and rear tires, respectively.

The $\theta_{v f}$ and $\theta_{v r}$ can be calculated using the following equations:

$$
\begin{aligned}
& \tan \left(\theta_{v f}\right)=\frac{V_{y}+l_{f} \dot{\psi}}{V_{x}}, \\
& \tan \left(\theta_{v r}\right)=\frac{V_{y}-l_{r} \dot{\psi}}{V_{x}} .
\end{aligned}
$$

The vehicle body side slip angle $\beta$ is one of the important parameters which gives the information about the chassis 


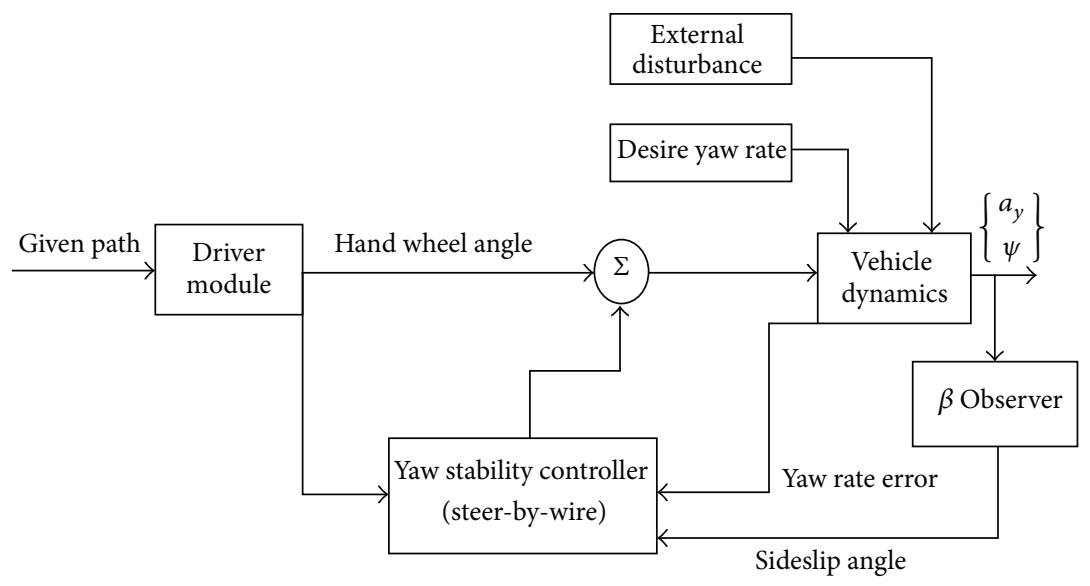

FIGURE 3: Block diagram of proposed yaw stability controller.

direction of the vehicle and it is calculated using the following equation:

$$
\beta=\arctan \left(\frac{V_{y}}{V_{x}}\right) .
$$

The rate of desired yaw orientation or yaw rate is defined as follows:

$$
\dot{\psi}_{\mathrm{des}}=\frac{V_{x}}{l_{f}+l_{r}} \delta .
$$

From the above equation, it can be noticed that the desired yaw rate depends on steering angle given by the driver, speed of the vehicle. Desired yaw rate provides the expected vehicle path information; however, due to external disturbance such as cross wind, asymmetric friction coefficients on front, and rear tires, there exists an error in the yaw rate and it can be calculated using the following equation:

$$
e_{r}=\dot{\psi}-\dot{\psi}_{\mathrm{des}}
$$

In order to analyze the above vehicle dynamics in time domain, a state space model for linear bicycle model with $x=\left[\begin{array}{ll}\beta & \dot{\psi}\end{array}\right]^{T}, y=\left[\begin{array}{ll}a_{y} & \dot{\psi}\end{array}\right]^{T}$ is used for analyzing yaw rate of the vehicle as given below:

$$
\begin{aligned}
{\left[\begin{array}{c}
\dot{\beta} \\
\ddot{\psi}
\end{array}\right]=} & {\left[\begin{array}{cc}
\frac{-C_{a f}-C_{a r}}{m v} & \frac{-C_{a f} l_{f}+C_{a r} l_{r}}{m v^{2}}-1 \\
\frac{C_{a r} l_{r}-C_{a f} l_{f}}{I_{z}} & \frac{-C_{a f} l_{f}^{2}-C_{a r} l_{r}^{2}}{I_{z} v}
\end{array}\right]\left[\begin{array}{l}
\beta \\
\dot{\psi}
\end{array}\right] } \\
& +\left[\begin{array}{c}
\frac{C_{a f}}{m v} \\
\frac{C_{a f} l_{f}}{I_{z}}
\end{array}\right] \delta+\left[\begin{array}{c}
\frac{1}{m v l} \\
\frac{1}{I_{z}}
\end{array}\right] M_{d},
\end{aligned}
$$

$$
\begin{aligned}
{\left[\begin{array}{c}
a_{y} \\
\dot{\psi}
\end{array}\right]=} & {\left[\begin{array}{cc}
-C_{a f}-C_{a r} & \frac{-C_{a f} l_{f}+C_{a r} l_{r}}{m} \\
0 & \frac{m v}{1}
\end{array}\right]\left[\begin{array}{c}
\beta \\
\dot{\psi}
\end{array}\right] } \\
& +\left[\begin{array}{c}
\frac{C_{a f}}{m} \\
0
\end{array}\right] \delta .
\end{aligned}
$$

\section{Control System Design}

The construction of proposed control system is shown in Figure 3. As the desired path of the vehicle depends upon the steering angle, yaw rate error, and side slip angle, these three parameters are taken as inputs to the proposed control system. Measurement of side slip angle is a difficult task; hence, an observer which uses a kalman filter is used in the present work. The steer-by-wire system receives the yaw rate of a vehicle using a yaw rate sensor and yaw rate error is calculated using the actual vehicle model. A correction in the steering angle is estimated using the fuzzy logic control system, which will be applied to the front wheels of the vehicle for maintaining the desired path of the vehicle.

The observer model to estimate the side slip angle of the vehicle using Kalman filter is discussed in the next section. The description about the fuzzy control system, such as fuzzification, fuzzy rules and inference, defuzzification is discussed in the subsequent sub sections.

3.1. Sideslip Angle Observer. Sideslip angle is an important parameter which is useful for determining the actual stability condition of a vehicle. In this work, a linear observer is used for estimating the sideslip angle because sensors for measuring side slip angle are more expensive. A model based Kalman filter is used for estimating the sideslip angle value using lateral acceleration and yaw rate has input as given in (9). The state equations for the observer are given below:

$$
\dot{\hat{x}}=A \hat{x}+B u-K(\hat{y}-y) .
$$




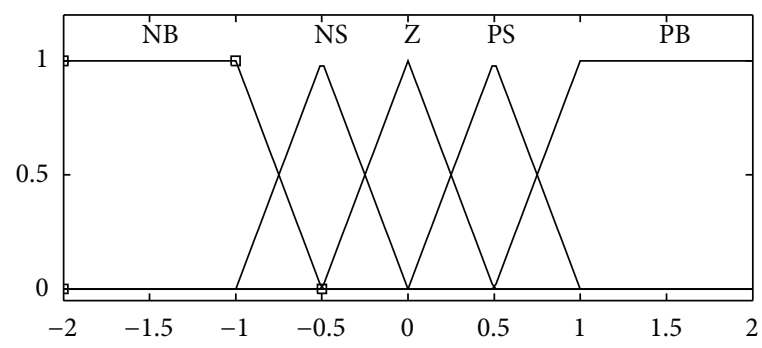

(a) Steering angle

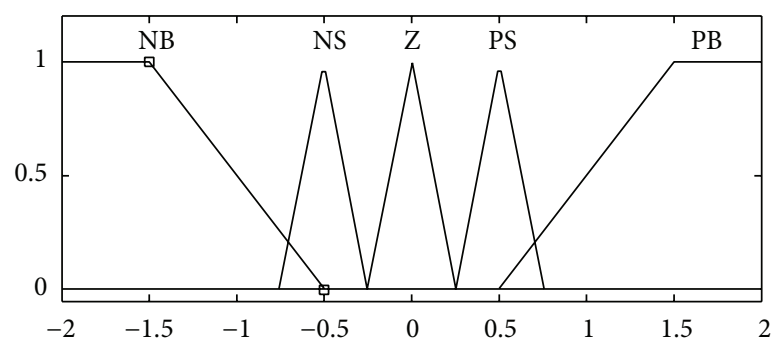

(b) Yaw rate error

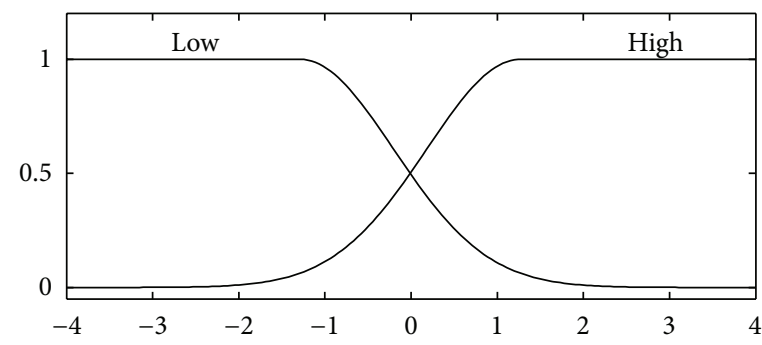

(c) Side slip angle

FIgURE 4: Membership functions for input variables.

The output of the observer is given by the following equation:

$$
\widehat{x}=\left[\begin{array}{c}
\widehat{\beta} \\
\widehat{\psi}
\end{array}\right] \quad y=\left[\begin{array}{c}
a_{y} \\
\dot{\psi}
\end{array}\right] .
$$

The model for determining Kalman gain $(K)$ [16] is shown below:

$$
K=\left[\begin{array}{cc}
\frac{\left[C_{a f} l_{f}-C_{a r} l_{r}\right] 2\left(\lambda_{1} \lambda_{2}\right) I_{z}}{\left(4 C_{a f} C_{a r}\right)\left(l^{2}\right)}-1 & \frac{1}{v} \\
-\lambda_{1}-\lambda_{2} & \frac{m\left[C_{a f} l_{f}^{2}+C_{a r} l_{r}^{2}\right]}{\left[C_{a f} l_{f}-C_{a r} l_{r}\right] I_{z}}
\end{array}\right],
$$

in which $\lambda_{1}$ and $\lambda_{2}$ are the assigned pole values of the observer.

3.2. Fuzzification. Proposed fuzzy control system with three inputs and one output is developed. The input variable, side slip angle is fuzzified to have two fuzzy sets, namely, Low and High. The steering angle and yaw rate error are fuzzified into five fuzzy sets: negative big (NB), negative small (NS), zero (Z), positive small (PS), and positive big (PB). The output variable, steering correction angle is fuzzified to have eleven fuzzy sets: (NB, NMH, NM, NMS, NS, Z, PS, PMS, PM, PMH, PB), where NMH and $\mathrm{PMH}$ represent Negative Medium High and Positive Medium High. NMS and PMS represent Negative Medium Small, Positive Medium Small. NM and PM represent Negative Medium and Positive Medium. Membership functions for the input and output variables of the fuzzy control system are shown in Figures 4 and 5 , respectively.

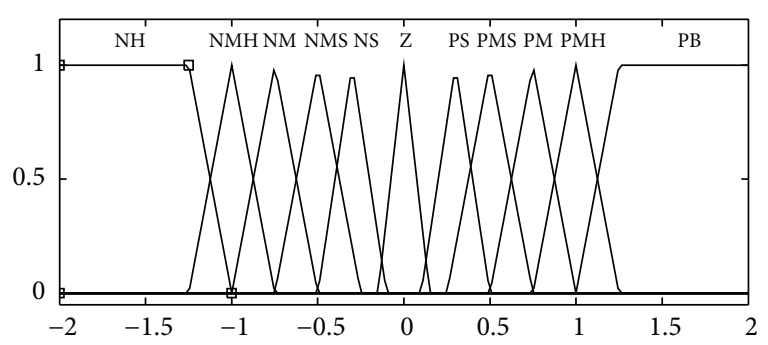

FIGURE 5: Membership functions for the output variable: correction angle.

3.3. Fuzzy Rules. For the given input values of steering angle, side slip angle, and yaw rate error, fuzzy rules were formulated for inferring the output correction angle to the front wheel using steer-by-wire system. By using fuzzy theory, proposed fuzzy control system uses Mamdani fuzzy inference system, which is characterized by the following fuzzy rules as shown in Table 1 .

\section{Simulation Results and Analysis}

Performance of the proposed fuzzy control system is compared with the existing fuzzy control system, which uses only two inputs such as steering angle of the front wheel and yaw rate error [15]. A simulation study was conducted using Simulink in Matlab for sinusoidal and step steering maneuver (in Figure 6) with the external disturbance (in Figure 7), which can be caused by asymmetric friction coefficients at the left and right tires. The simulation results are presented for the different vehicle speeds such as $10 \mathrm{~m} / \mathrm{s}$ and $30 \mathrm{~m} / \mathrm{s}$. The vehicle 


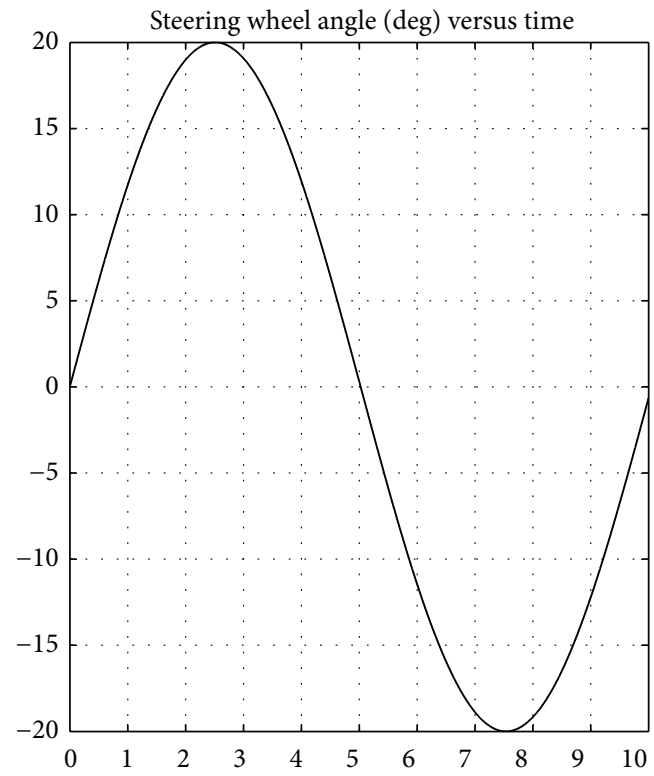

(a) Sinusoidal input

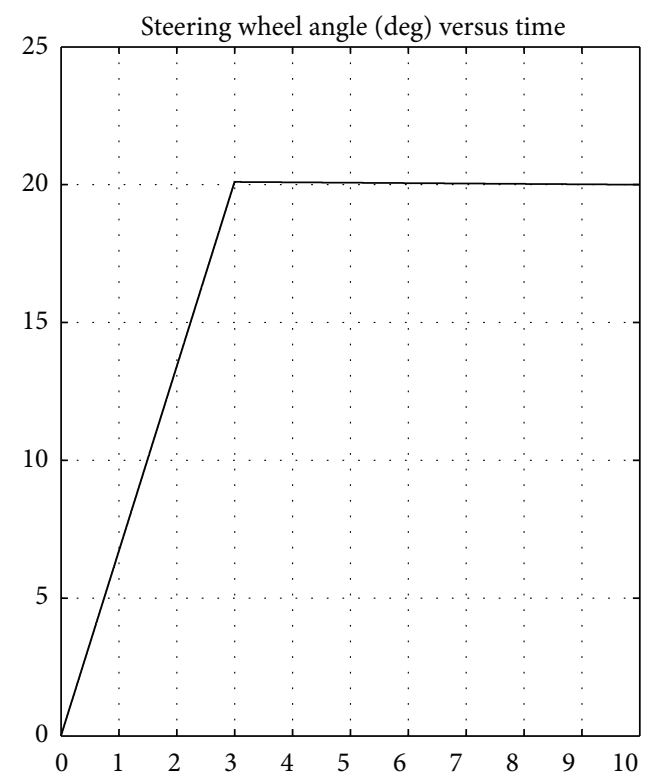

(b) Step input

FIgURE 6: Steering inputs.

parameters used for the computer simulations are given in Table 2.

4.1. Sinusoidal Maneuver. A sinusoidal steering input can be associated with a slalom test, which is performed for assessing the yaw roll stability of a vehicle. Figures 8 and 9 show the comparison of vehicle responses for the yaw rate and yaw rate error of the vehicle during sinusoidal maneuver. It is observed that the yaw rate error and amplitude of disturbance are much lesser for the proposed control system than the existing model and uncontrolled vehicle. The proposed fuzzy controller

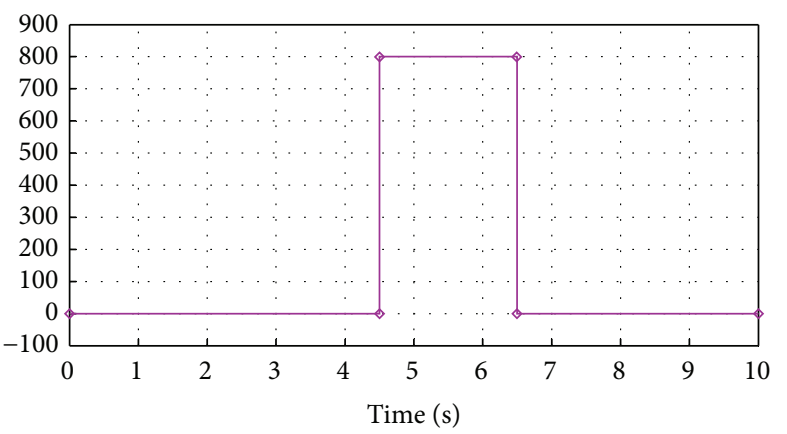

FIGURE 7: External disturbance.

TABLE 1: Fuzzy interference rule.

\begin{tabular}{lcccccc}
\hline $\begin{array}{l}\text { Side slip } \\
\text { angle }\end{array}$ & \multirow{2}{*}{ Steering angle } & \multicolumn{5}{c}{ Yaw rate error } \\
& & NB & NS & Z & PS & PB \\
\hline \multirow{4}{*}{ Low } & NB & NS & NS & Z & PB & PB \\
& NS & NMS & NMS & Z & PMH & PMH \\
& Z & NM & NM & Z & PM & PM \\
& PS & NMH & NMH & Z & PMS & PMS \\
& PB & NH & NH & Z & PS & PS \\
\hline \multirow{4}{*}{ High } & NB & NH & NH & Z & PS & PS \\
& NS & NMH & NMH & Z & PMS & PMS \\
& Z & NM & NM & NS & PMS & PMS \\
& PS & NMS & NMS & NS & NS & NS \\
& PB & NS & NS & NS & NS & NS \\
\hline
\end{tabular}

TABLE 2: Vehicle parameters.

\begin{tabular}{lc}
\hline Vehicle mass $(m)$ & $1663 \mathrm{Kg}$ \\
Yaw moment of inertia $\left(I_{z}\right)$ & $2704 \mathrm{Kgm}^{2}$ \\
Distance from front axle to CG $\left(l_{f}\right)$ & $1.1437 \mathrm{~m}$ \\
Distance from rear axle to CG $\left(l_{r}\right)$ & $1.431 \mathrm{~m}$ \\
Wheel base $(l)$ & $2.56 \mathrm{~m}$ \\
Front tyre cornering stiffness $\left(C_{a f}\right)$ & $63946 \mathrm{~N} / \mathrm{rad}$ \\
Rear tyre cornering stiffness $\left(C_{a r}\right)$ & $55040 \mathrm{~N} / \mathrm{rad}$ \\
Vehicle speed $(v)$ & $10 \mathrm{~m} / \mathrm{s}$ and $30 \mathrm{~m} / \mathrm{s}$ \\
\hline
\end{tabular}

was performing better in both low speed and high speed. It means that vehicle with the proposed controlled system follows the desired path much closer than the uncontrolled vehicle.

4.2. Step Maneuver. The step input has been used to simulate conditions similar to the straight line cornering test. Responses for the yaw rate, side slip angle, and yaw rate error of proposed model are compared with the existing approach and uncontrolled vehicles for step steering input as shown in Figures 10 and 11. As in the previous case of sinusoidal steering input, the vehicle with the proposed control system behaves in a stable manner at low speed and high speed with 

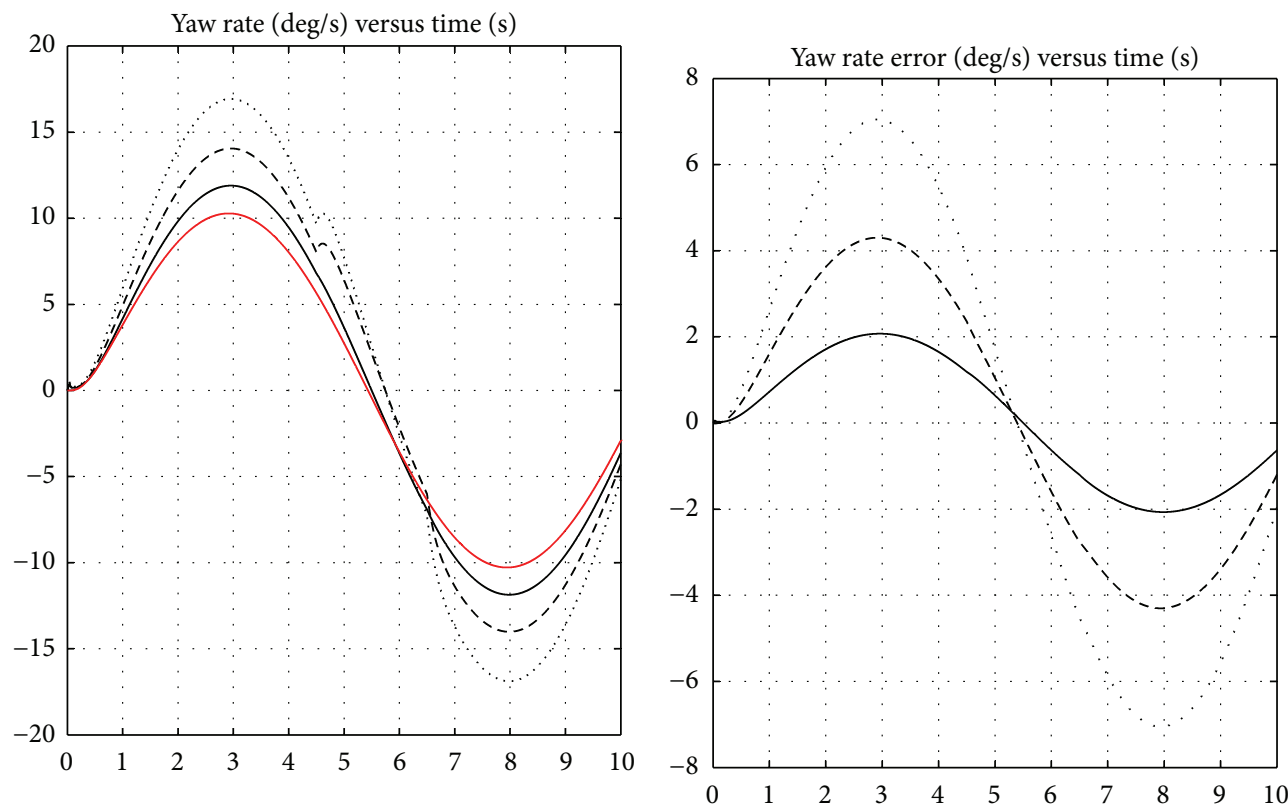

Proposed approach/1

- - - Existing approach/1

Without controller/1

_ Proposed approach $/ 3$

- - Existing approach $/ 3$

...... Without controller/3

(a) Yaw rate

(b) Yaw rate error

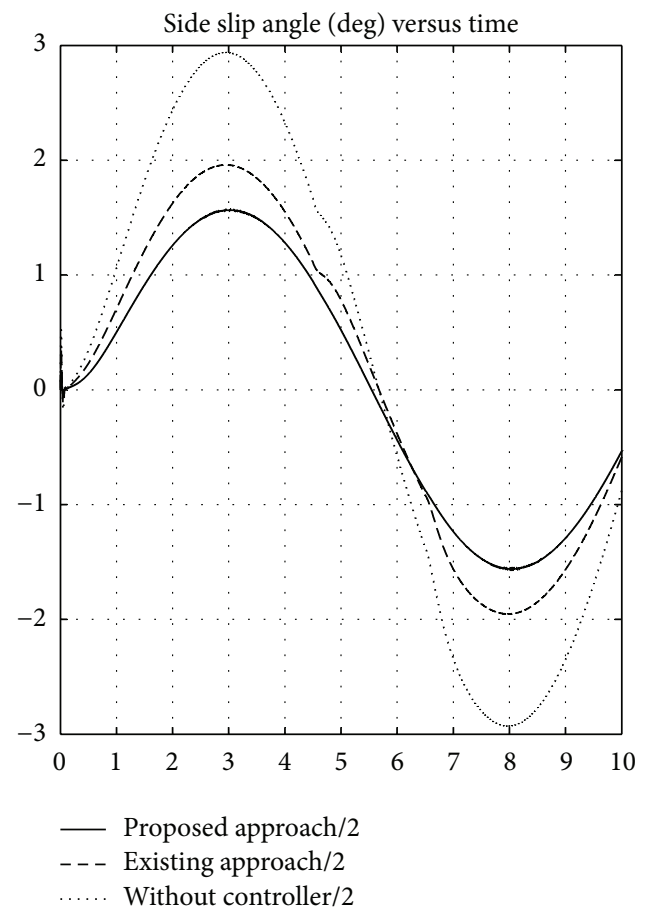

(c) Estimated sideslip angle

FIGURE 8: Vehicle response for sinusoidal input with external disturbance for $10 \mathrm{~m} / \mathrm{s}$. 


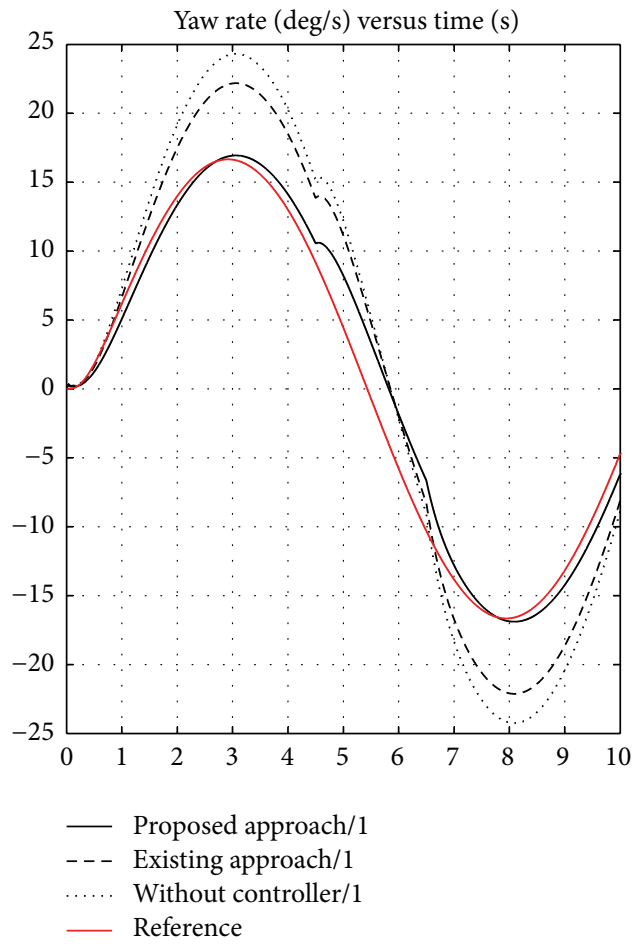

(a) Yaw rate
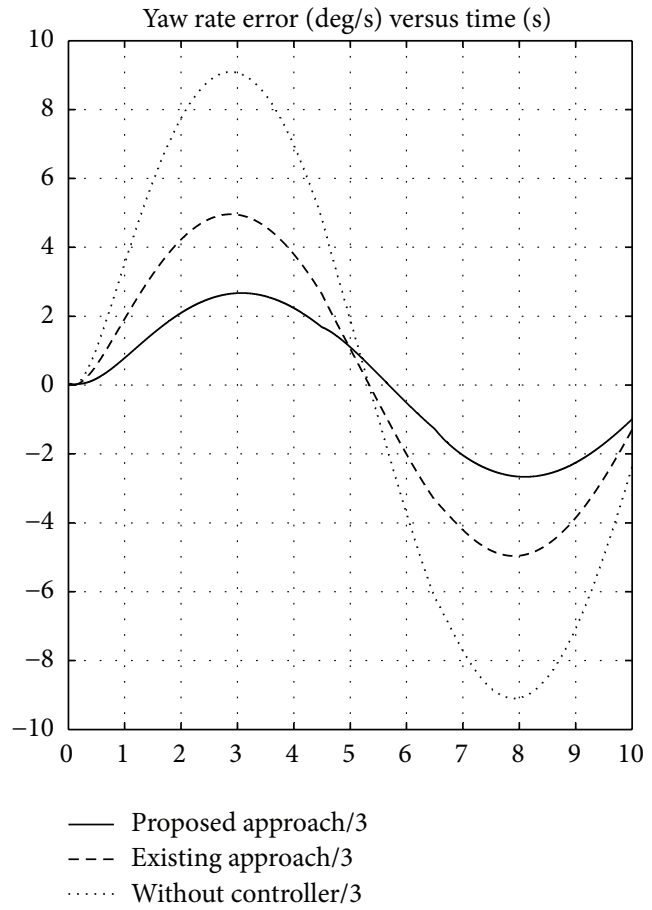

(b) Yaw rate error

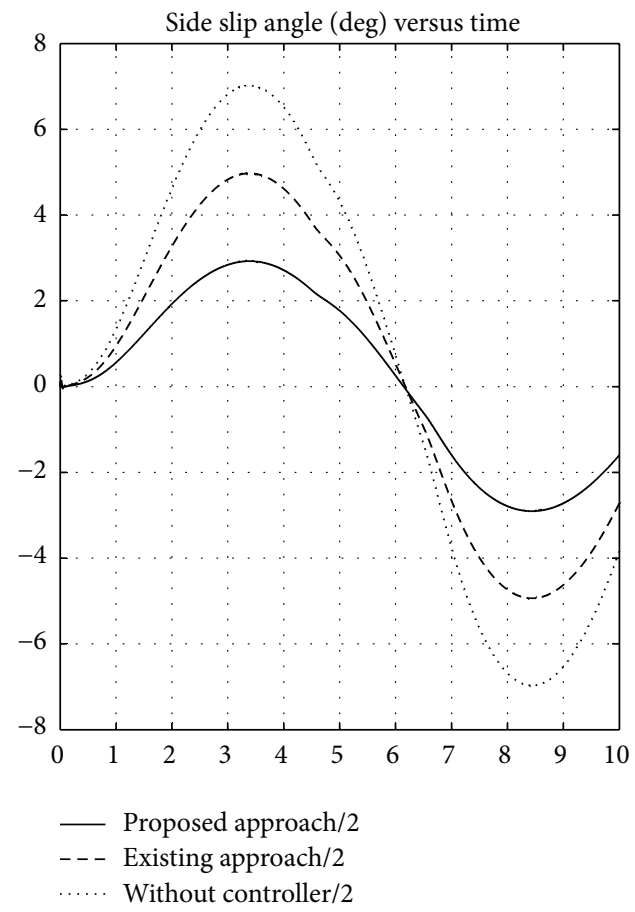

(c) Estimated sideslip angle

FIGURE 9: Vehicle response for sinusoidal input with external disturbance for $30 \mathrm{~m} / \mathrm{s}$.

low yaw rate error and effect of disturbance as compared with the uncontrolled vehicle. Also the responses to yaw rate of vehicle with the proposed control system is closer to reference as shown in Figure 11(a). 


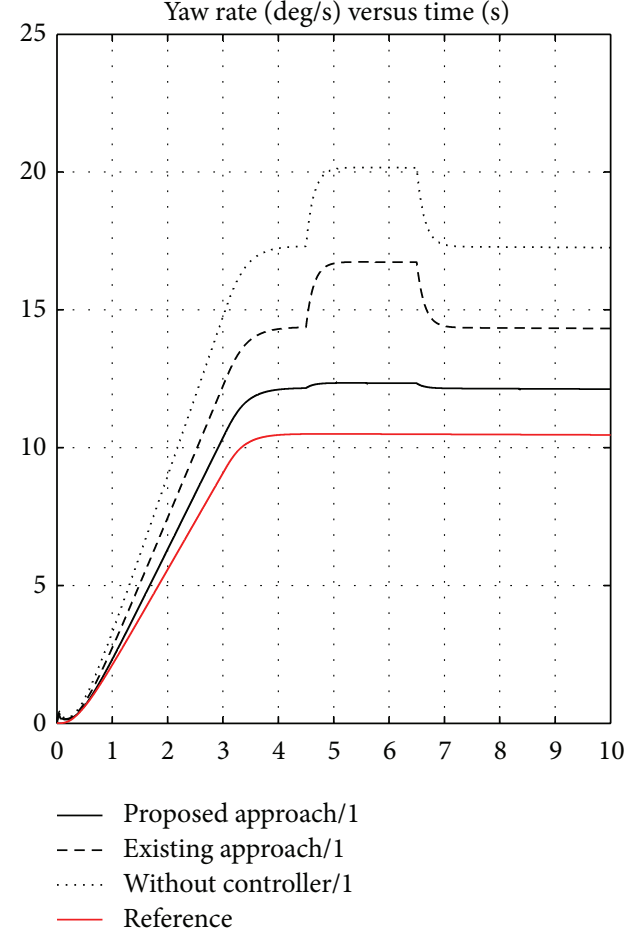

(a) Yaw rate

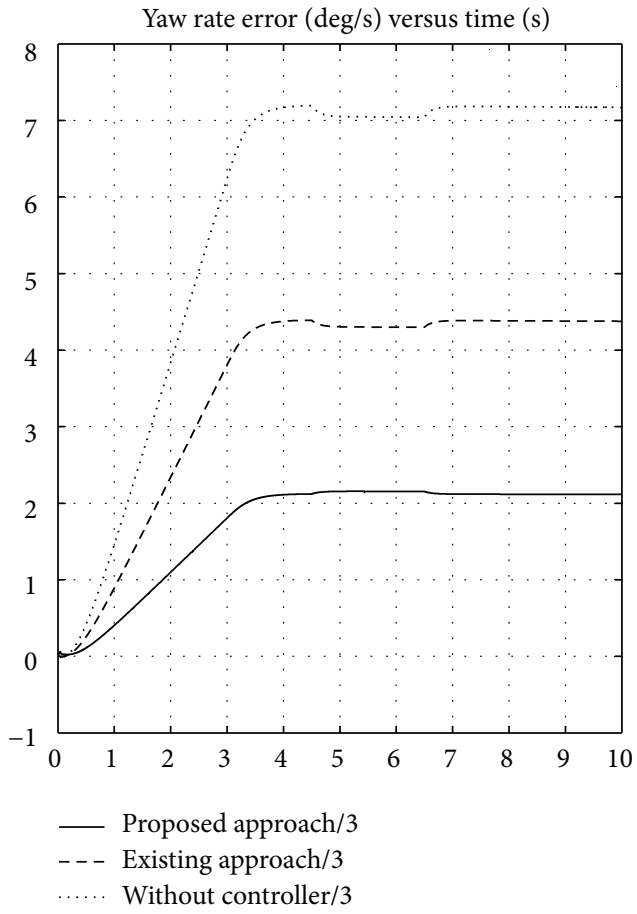

(b) Yaw rate error

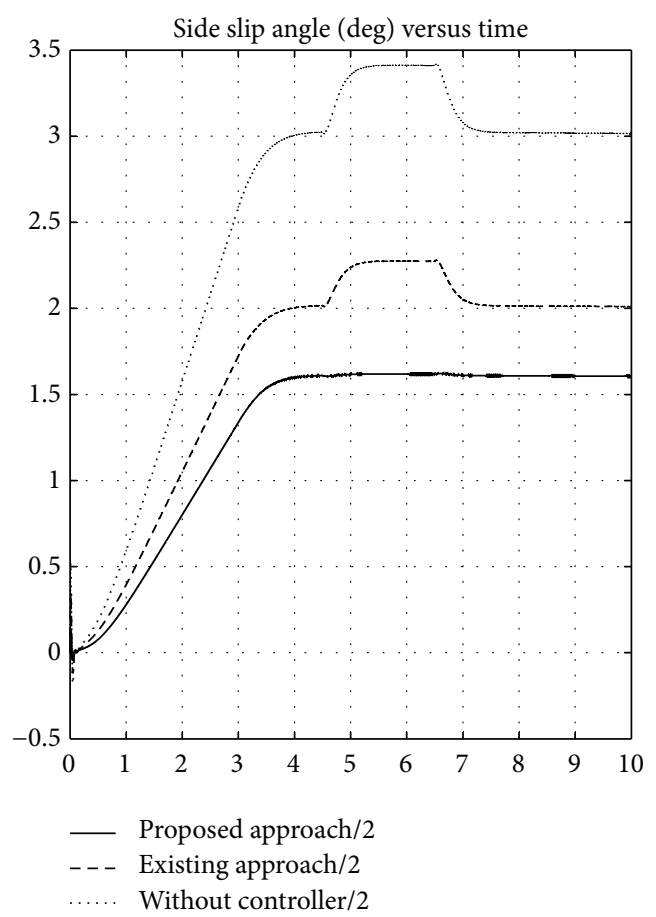

(c) Estimated sideslip angle

FIGURE 10: Vehicle response for step input with external disturbance for $10 \mathrm{~m} / \mathrm{s}$.

\section{Conclusion}

This paper proposes a fuzzy logic based yaw stability controller for active front steering of the vehicle. Proposed control system takes steering angle, yaw rate error, side slip angle as inputs and estimates the additional steering angle to be applied to the front wheel of the vehicle for maintaining yaw stability of the vehicle. Mathematical model for the lateral dynamics of vehicle is derived for determining the yaw rate error of the vehicle. A linear observer model is used for 


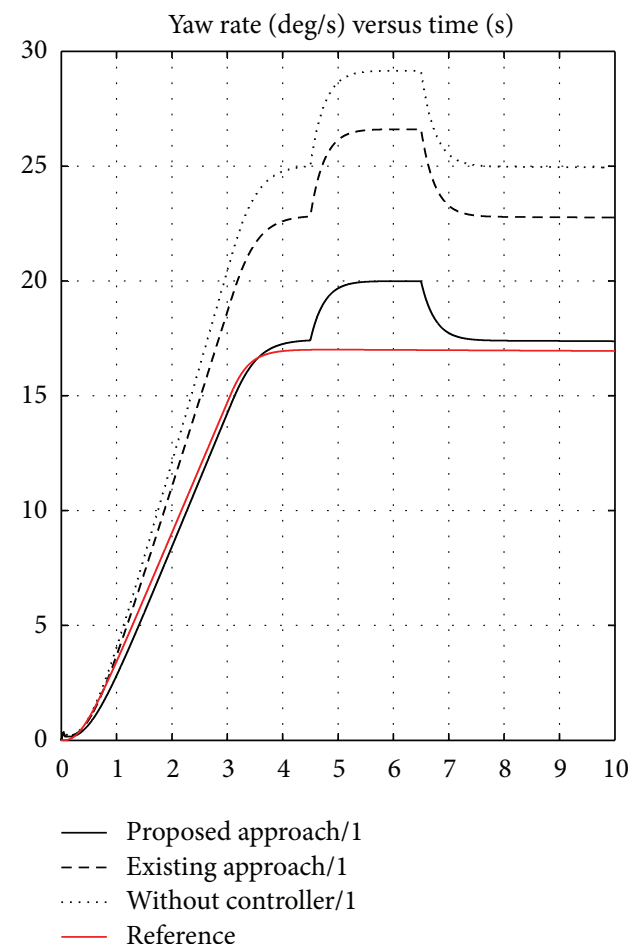

(a) Yaw rate

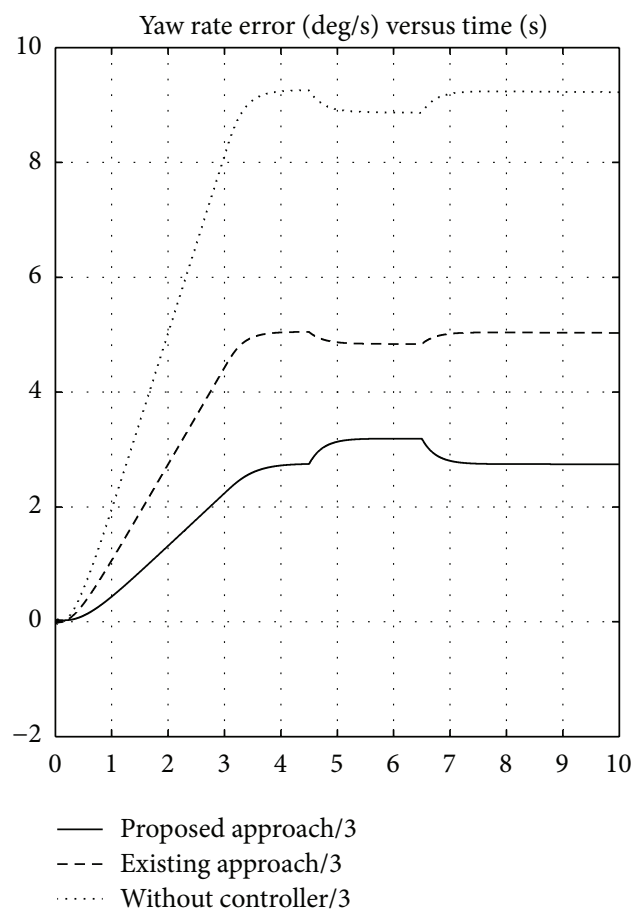

(b) Yaw rate error

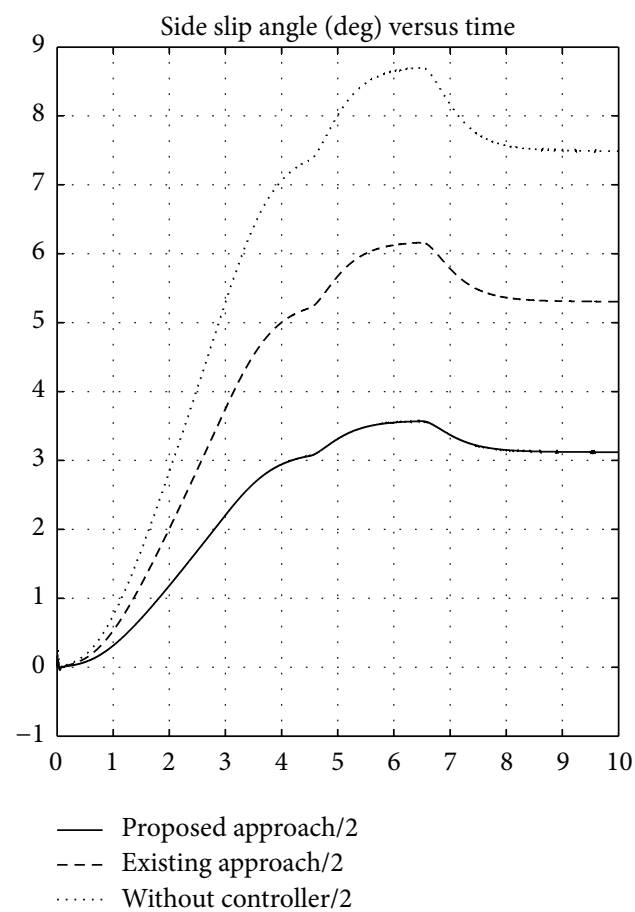

(c) Estimated sideslip angle

FIGURE 11: Vehicle response for step input with external disturbance for $30 \mathrm{~m} / \mathrm{s}$.

estimating the side slip angle of the vehicle. The performance of the proposed fuzzy based yaw stability control system has been evaluated using computer simulations with sinusoidal and step input maneuver. Simulation results show that the proposed system has smaller yaw rate error and maintain the stability of the vehicle under disturbance condition also, as compared with the existing model and uncontrolled vehicle. 


\section{Nomenclature}

$a_{y}:$ Lateral acceleration

$F_{y f}$ : Lateral tire force on front wheels

$V_{x}$ : Longitudinal velocity

$F_{y r}$ : Lateral tire force on rear wheels

$V_{y}$ : Lateral velocity

$l_{f}$ : Longitudinal distance from CG to front tires

$m$ : Mass of the vehicle

$l_{r}$ : Longitudinal distance from CG to rear tires

$I_{z}$ : Yaw moment of inertia of vehicle

$C_{a f}$ : Cornering stiffness of front tires

$\psi: \quad$ Yaw angle of vehicle

$C_{a r}$ : Cornering stiffness of rear tires

$\dot{\psi}$ : Yaw velocity of vehicle

$\theta_{v f}$ : Velocity angle of front tires

$\delta$ : $\quad$ Road wheel angle

$\theta_{v r}$ : Velocity angle of rear tires

$M_{d}$ : External Disturbance.

\section{Conflict of Interests}

The authors declare that there is no conflict of interests regarding the publication of the paper.

\section{References}

[1] J. Ackermann, "Robust decoupling, ideal steering dynamics and yaw stabilization of 4WS cars," Automatica, vol. 30, no. 11, pp. 1761-1768, 1994.

[2] J. Ackermann and T. Bünte, "Yaw disturbance attenuation by robust decoupling of car steering," Control Engineering Practice, vol. 5, no. 8, pp. 1131-1136, 1997.

[3] B. A. Güenç, L. Güvenç, and S. Karaman, "Robust yaw stability controller design and hardware-in-the-loop testing for a road vehicle," IEEE Transactions on Vehicular Technology, vol. 58, no. 2, pp. 555-571, 2009.

[4] M. Canale, L. Fagiano, A. Ferrara, and C. Vecchio, "Vehicle yaw control via second-order sliding-mode technique," IEEE Transactions on Industrial Electronics, vol. 55, no. 11, pp. 39083916, 2008.

[5] P. Falcone, F. Borrelli, J. Asgari, H. E. Tseng, and D. Hrovat, "Predictive active steering control for autonomous vehicle systems," IEEE Transactions on Control Systems Technology, vol. 15, no. 3, pp. 566-580, 2007.

[6] S. Oncu, S. Karaman, L. Guvenc et al., "Robust yaw stability controller design for a light commercial vehicle using a hardware in the loop steering test rig," in Proceedings of the IEEE Intelligent Vehicles Symposium, pp. 852-859, June 2007.

[7] K. Nam, S. OH, H. Fujimoto, and Y. Hori, "Robust yaw stability control for electric vehicles based on active front steering control through a steer-by-wire system," International Journal of Automotive Technology, vol. 13, no. 7, pp. 1169-1176, 2012.

[8] D. Bianchi, A. Borri, M. D. Di Benedetto, S. Di Gennaro, and G. Burgio, "Adaptive integrated vehicle control using active front steering and rear torque vectoring," International Journal of Vehicle Autonomous Systems, vol. 8, no. 2-4, pp. 85-105, 2010.

[9] S. Arabi and M. Behroozi, "Design of an integrated active front steering and active rear differential controller using fuzzy logic control," in Proceedings of the World Congress on Engineering (WCE '10), vol. 2, pp. 1409-1414, July 2010.

[10] A. Hakima and S. Ameli, "Improvement of vehicle handling by an integrated control system of four wheel steering and ESP with fuzzy logic approach," in Proceedings of the 2nd International Conference on Mechanical and Electrical Technology (ICMET '10), pp. 738-744, September 2010.

[11] B. L. Boada, M. J. L. Boada, and V. Díaz, "Fuzzy-logic applied to yaw moment control for vehicle stability," Vehicle System Dynamics, vol. 43, no. 10, pp. 753-770, 2005.

[12] M. Sharif-Ul Hasan and S. Anwar, "Yaw stability control system for an automobile via steer-by-wire," in Proceedings of the 4th International Conference on Electrical and Computer Engineering (ICECE '06), pp. 345-348, December 2006.

[13] S.-J. Ko, J.-J. Kim, and J.-J. Lee, "Yaw stabilization of a vehicle by yaw stability controller based on fuzzy logic," in Proceedings of the 8th Symposium on Advanced Intelligent System (ISIS '07), Sokcho-city, Republic of Korea, 2007.

[14] A. Goodarzi, S. Arabi, and E. Esmailzadeh, "Design of an integrated AFS/ACD control system to enhance 4WD vehicles dynamics and stability," in Proceedings of the International Design Engineering Technical Conferences and Computers and Information in Engineering Conference (IDETC/CIE '10), pp. 307-314, can, August 2010.

[15] S. Ghosh, A. Deb, M. Mahala, M. Tanbakuchi, and M. Makowski, "Active yaw control of a vehicle using fuzzy logic algorithm," SAE International, 2012-01-0229.

[16] C. Geng, T. Uchida, and Y. Hori, "Body slip angle estimation and control for electric vehicle with in-wheel motors," in Proceedings of the 33rd Annual Conference of the IEEE Industrial Electronics Society (IECON '07), pp. 351-355, November 2007. 

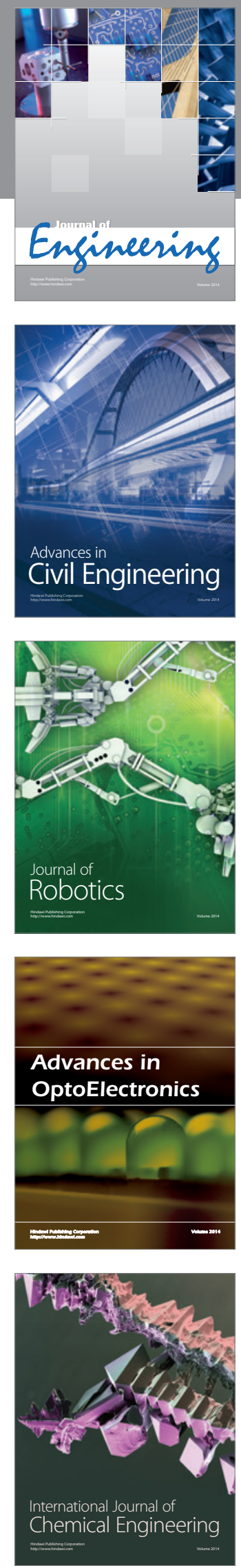

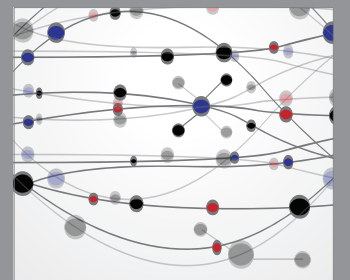

The Scientific World Journal
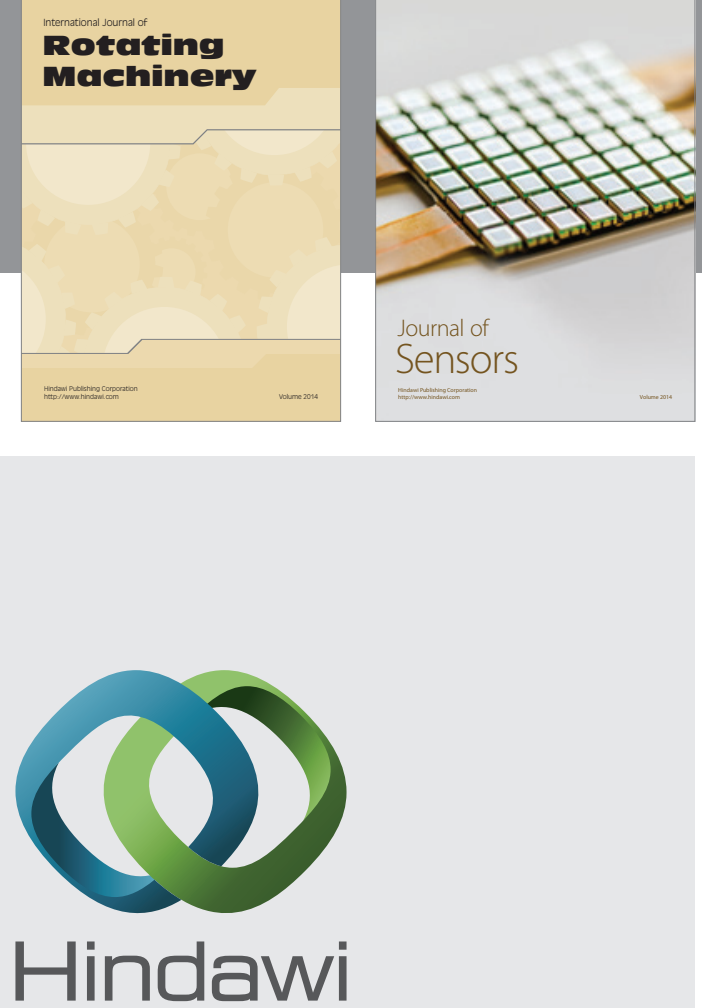

Submit your manuscripts at http://www.hindawi.com
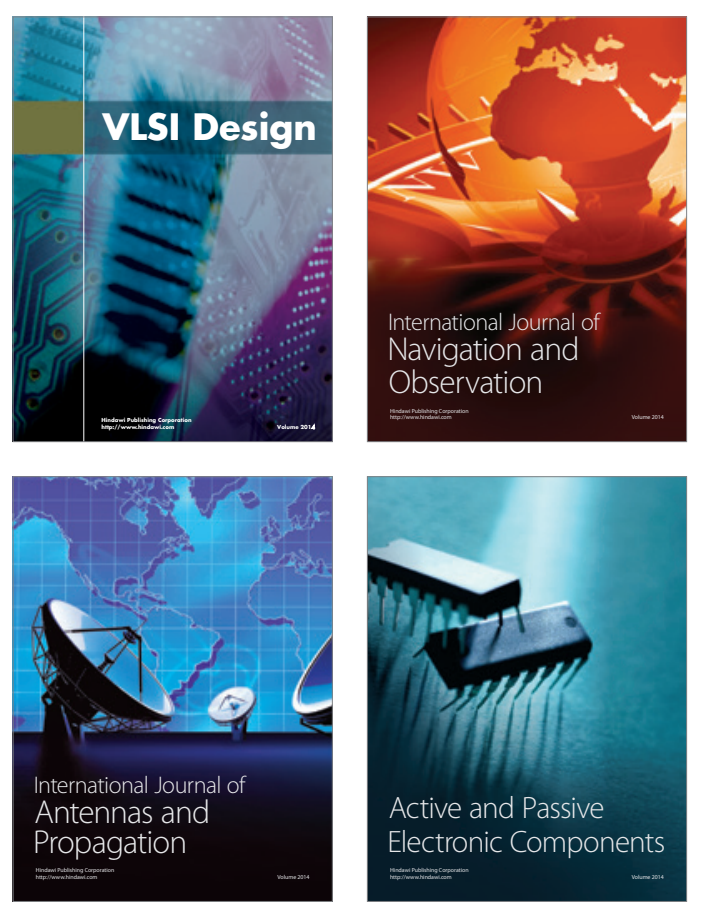
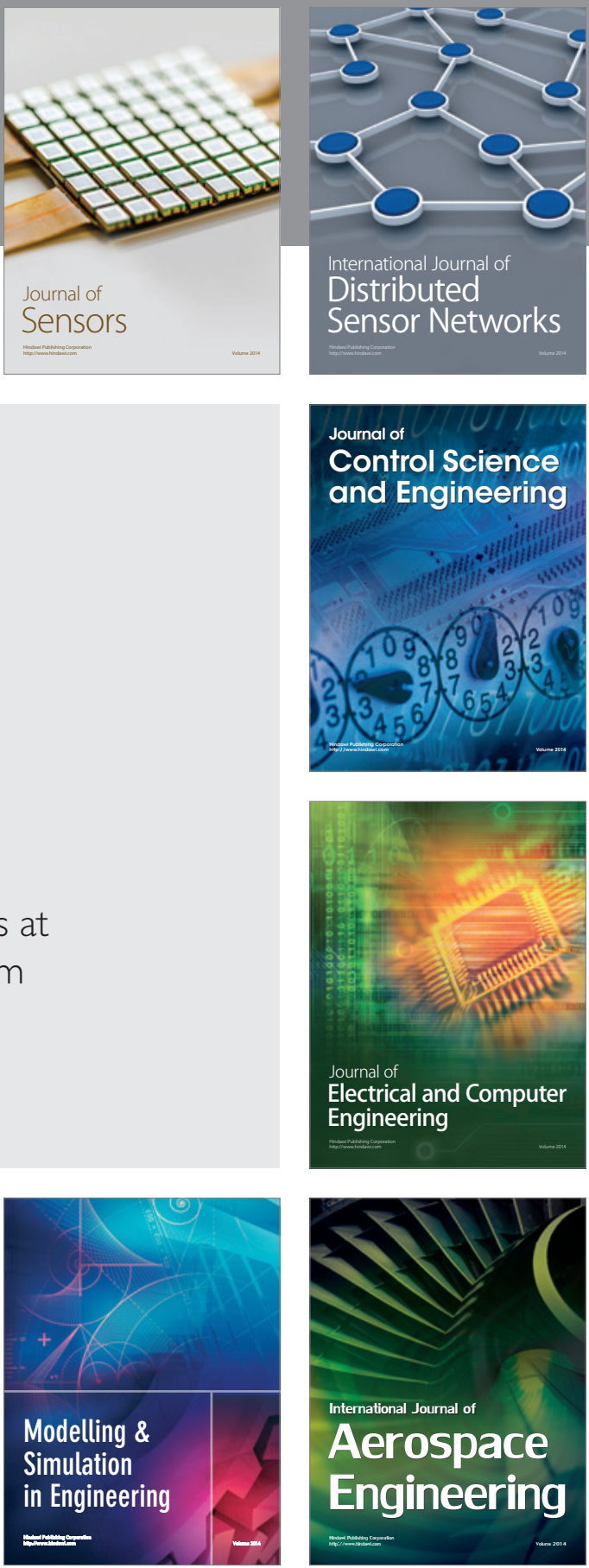

Journal of

Control Science

and Engineering
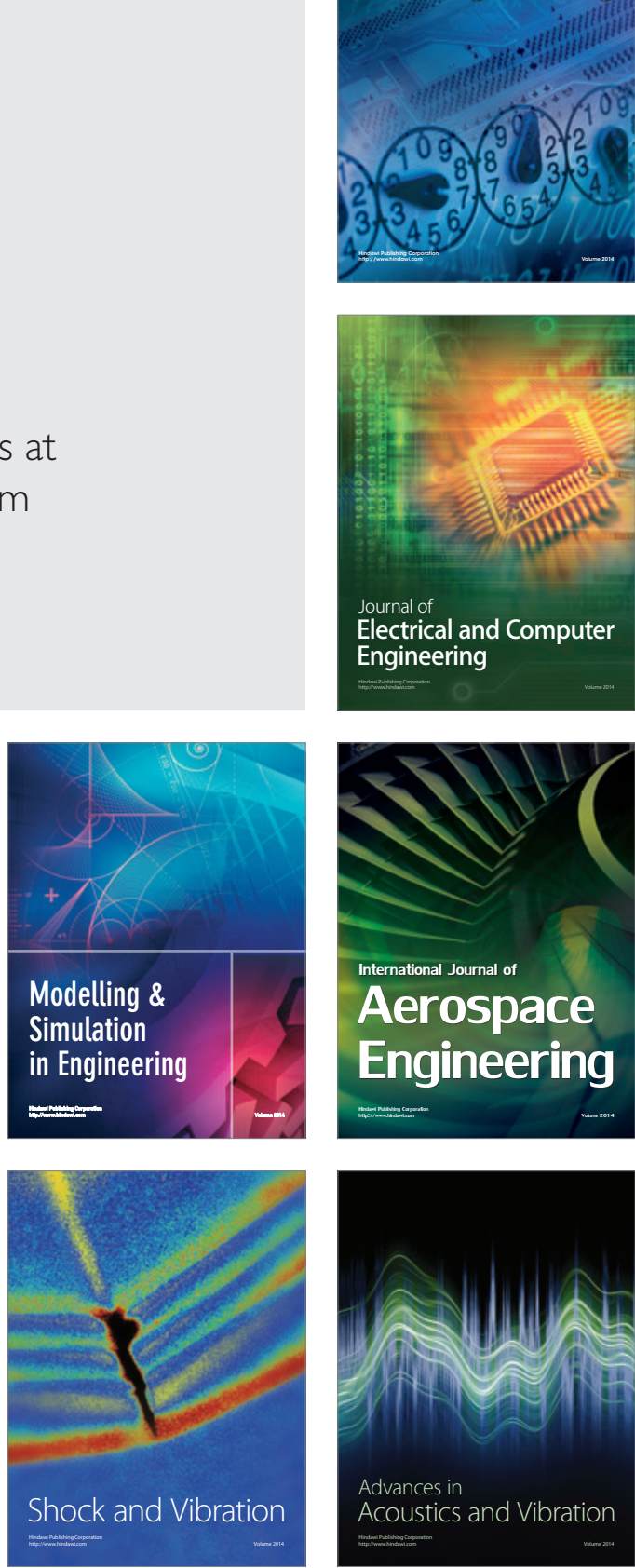